\title{
Diurnal Periodicity of the Expression of Genes Involved in Monolignol Biosynthesis in Differentiating Xylem of Cryptomeria japonica
}

\author{
Saori Sato, Masato Yoshida, Yousuke Ashizaki, Hiroyuki Yamamoto \\ Laboratory of Bio-Material Physics, Graduate School of Bioagricultural Sciences, Nagoya University, Nagoya, Japan \\ Email: yoshida@agr.nagoya-u.ac.jp
}

How to cite this paper: Sato, S., Yoshida, M., Ashizaki, Y. and Yamamoto, H. (2016) Diurnal Periodicity of the Expression of Genes Involved in Monolignol Biosynthesis in Differentiating Xylem of Cryptomeria japonica. American Journal of Plant Sciences, 7, 2457-2469.

http://dx.doi.org/10.4236/ajps.2016.717214

Received: November 15, 2016

Accepted: December 10, 2016

Published: December 13, 2016

Copyright $\odot 2016$ by authors and Scientific Research Publishing Inc. This work is licensed under the Creative Commons Attribution International License (CC BY 4.0).

http://creativecommons.org/licenses/by/4.0/

\begin{abstract}
The cell wall in wood is mainly composed of three components: cellulose, hemicellulose and lignin. According to electron microscopy observations of the innermost surface of cell walls in the tracheids of Cryptomeria japonica, cellulose microfibrils are deposited during the day and a matrix containing hemicellulose is deposited at night. This indicates that the deposition of cell wall components exhibits diurnal periodicity. To gain new insights into the diurnal periodicity of lignin deposition not revealed by microscopic observations, we examined diurnal fluctuations in the expression of genes involved in monolignol biosynthesis in $C$. japonica saplings grown in the field and in growth chambers under a $12 \mathrm{~h}$ light/dark cycle. In the field experiment, two gene expression peaks were observed daily, at dusk and dawn. In the growth chamber experiment, two daily peaks were observed $0 \mathrm{~h}$ and $6-9 \mathrm{~h}$ after the light switched on.
\end{abstract}

\section{Keywords}

Cryptomeria japonica, Diurnal Periodicity, Monolignol, Secondary Wall, Tracheid

\section{Introduction}

Cell walls in wood are mainly composed of three components: cellulose, hemicellulose, and lignin. Cellulose is a linear polymer composed of glucose residues. Its molecular chain is elongated by cellulose-synthesizing complexes embedded in the plasma membrane [1]. Synthesized cellulose chains are bundled to form fibers called cellulose microfibrils, which are deposited appositionally along the innermost surface of cell walls [2]. Hemicellulose is a branched polysaccharide composed of sugars such as glucose, xylose, and mannose, and it is synthesized in the Golgi apparatus. Newly synthesized 
hemicellulose is transported to the plasma membrane by Golgi vesicles, secreted to the innermost surface of the cell walls via vesicle integration into the plasma membrane, and deposited in the walls [3]. Lignin is a net-like polymer composed of phenylpropane units called monolignols, which are synthesized in the cytoplasm and transported to the cell wall, where they are polymerized by peroxidase and laccase [4] [5].

After cell wall components are synthesized in the cytoplasm, they are transported out of the plasma membrane and deposited; hence, cell walls are basically formed from the outer side, inward. Therefore, when making a longitudinal section of differentiating xylem and observing the innermost surface of the cell wall by microscopy, the component that has been synthesized and deposited most recently should be visible. Observations of the innermost surfaces of cell walls have been observed with electron microscopy in Populusnigra, Cryptomeria japonica, and others [6] [7]. For instance, when the innermost surfaces of tracheids during the thickening stage of the middle layer of the secondary cell walls (S2 layer) were observed in $C$. japonica grown in the field or in a growth chamber, cellulose microfibrils were evident during the day or the light period, and a matrix covering the microfibrils was evident at night or during the dark period. These observations led to the conclusion that the deposition of cell wall components has diurnal periodicity [8] [9].

Immunostaining with antibodies that specifically label hemicellulose has been used to determine the composition of the matrix that deposits at night. In experiments on $C$. japonica saplings, the matrix observed at night contained glucomannan and xylan, which are the main components of hemicellulose in conifers [7] [10]. Bobák and Nečesaný [6] observed that the matrix in the innermost surfaces of cell walls was composed of lignin. However, during thickening of the S2 layer, lignin is deposited in the primary walls and the outer layer of the secondary walls, both of which exist outside this layer [11] [12]; therefore, it is unlikely that lignin exists in the innermost surfaces of the S2 layer. Because lignin deposits intussusceptively from the outer towards the inner side of cell walls, it is difficult to determine the periodicity of lignin deposition using only observations of the innermost surface of the wall via electron microscopy.

Several studies have examined the diurnal periodicity of the expression of genes involved in the synthesis of cell wall components. For instance, Harmer et al. [13] grew Arabidopsis thaliana under constant light and examined the fluctuations of gene expression related to the phenylpropanoid biosynthetic pathway, and found that expression peaked before the subjective dawn. Solomon et al. [14] examined fluctuations in the expression of genes involved in lignin biosynthesis in Eucalyptus grandis $\times$ E. camaldulensis and E. grandis $\times$ E. urophylla grown in the field, and reported that expression peaked twice daily, once during the day and once before dawn.

We selected $C$. japonica as the experimental model for this study as it had been previously studied for cell wall component deposition in the innermost layer. We examined it for diurnal fluctuations in the expression of genes involved in the synthesis of cell wall components. Based on our results, we discussed the relationship between the diurnal periodicity of cellulose microfibril/matrix deposition in the innermost surface 
of the cell wall that was previously observed with microscopy, and the diurnal fluctuations in the expression of genes involved in the synthesis of cellulose and hemicellulose. In addition, we examined diurnal fluctuations of the expression of genes involved in monolignol biosynthesis to gain new insights into the diurnal periodicity of lignin deposition not previously revealed via microscopy.

\section{Materials and Methods}

\subsection{Plant Growth Conditions}

The experiments were conducted in two environments, in the field and in growth chambers. The field experiment was conducted from April to July 2015 in a field owned by Nagoya University, Japan. Sixteen 3-year-old $C$. japonica saplings $(\sim 110 \mathrm{~cm}$ in height, $\sim 15 \mathrm{~mm}$ in basal diameter) were planted in plastic pots filled with a mixture of red soil and compost. To maintain vertical stem growth, saplings were fixed loosely to stakes with wire. The growth chamber experiment was conducted from April to June 2014. Sixteen 2-year-old C. japonica saplings ( $\sim 64 \mathrm{~cm}$ in height, $\sim 13 \mathrm{~mm}$ in basal diameter) were planted in pots and fixed to stakes with wire. The saplings were grown in the field from April to May and moved to the growth chambers (MLR-350H, Sanyo, Japan) with the pots 1 week before sample collection. The temperature of the growth chambers was set to $25^{\circ} \mathrm{C}$, and the lighting was set to a $12 \mathrm{~h}$ light/dark cycle. In both the field and growth chamber experiments, meteorological equipment (TR-74Ui, T \& D Corporation, Nagano, Japan) was placed beside the saplings to measure temperature, illumination intensity, and ultraviolet intensity.

\subsection{Sampling Schedule}

In the field experiment, samples were taken from 29 June to 1 July 2015. Plants were sampled every $3 \mathrm{~h}$ (10:00, 13:00, 16:00, 19:00, 22:00, 01:00, 04:00, and 07:00). The eight obtained samples were compiled as one set, and totally two sets of xylem samples were collected in the field experiment. In the growth chamber experiment, samples were taken twice, on 30 May and 6 June. Plants were sampled 0, 3, 6, 9, 12, 15, 18, and $21 \mathrm{~h}$ after the light switched on. The eight obtained samples were compiled as one set, and totally two sets of xylem samples were collected in the growth chamber experiment.

\subsection{Collection of Differentiating Xylem and Quantification of Gene Expression}

After removing the bark, differentiating xylem tissue was scraped from the stems. The collected tissues were frozen immediately in liquid nitrogen and stored at $-80^{\circ} \mathrm{C}$ until further use. Total RNA was extracted from the tissues using an RNeasy Plant Mini Kit (Qiagen, Valencia, CA, USA) according to the manufacturer's protocol. The RNA samples were treated with DNase I (Takara Bio, Kusatsu, Japan) to digest contaminating genomic DNA. Conversion into cDNA was performed using Prime Script RT Master Mix (Takara Bio). The quantitative reactions were performed on a Step One Plus Real Time PCR System (Life Technologies, Carlsbad, CA, USA) using the POWER SYBR 
Green PCR Master Mix (Life Technologies). The reaction mixture $(20 \mu \mathrm{L})$ contained 2 $\times$ POWER SYBR Master Mix (Life Technologies), $0.2 \mu \mathrm{M}$ each of the forward and reverse primers, and $5 \mathrm{ng}$ of template CDNA. PCR was performed under the following conditions: $95^{\circ} \mathrm{C}$ for $10 \mathrm{~min}$, followed by 40 cycles at $95^{\circ} \mathrm{C}$ for $15 \mathrm{~s}$ and $58^{\circ} \mathrm{C}$ for $60 \mathrm{~s}$. Dissociation curve analyses and electrophoresis were performed after the PCR runs to confirm product specificity and to check for primer dimer production. Gene expression was normalized to cyclophilin as an internal reference gene. Relative gene expression was calculated using the $2^{-\Delta \Delta \mathrm{Ct}}$ method [15]. All of the reactions were performed in triplicate.

\subsection{Genes Examined and Primer Synthesis}

To assess genes involved in cellulose synthesis, we quantified the transcript abundance of two genes: cellulose synthase A (CesA) and sucrose synthase (SUS). To assess the genes involved in lignin synthesis, we quantified the transcript abundance of eight genes: phenylalanine ammonium lyase $(P A L)$, cinnamate 4-hydroxylase $(C 4 H)$, 4-coumarate coenzyme A ligase (4CL), p-hydroxycinnamoyl-CoA: quinate/shikimate-hydroxy cinnamoyltransferase $(H C T), p$-coumarate 3-hydroxylase $(C 3 H)$, caffeoyl-CoA O-methyltransferase (CCoAOMT), cinnamoyl-CoA reductase $(C C R)$, and cinnamyl alcohol dehydrogenase $(C A D)$. We followed the protocol used by Mishima et al. [16] to generate the primer sequences for these genes. To assess genes involved in hemicellulose synthesis, we quantified the transcript abundances of two genes required for glucomannan and xylan synthesis. From the expressed sequence tag database of $C$. japonica (Forest GEN, http://forestgen.ffpri.affrc.go.jp/ja/index.html), we searched for a $C$. japonica homolog of the cellulose synthase-like A (CslA) gene involved in the elongation of glucomannan main chains using a Pinustaeda homolog (PtaCslA1) [17] as the query. Similarly, we searched for a $C$. japonica homolog of the glycosyltransferase ( $G T$ ) gene involved in xylan main chain elongation using IRX10 from $A$. thaliana [18] [19] as the query. Based on the results (Cluster ID: CJC19320, CJC03090), primer sequences were designed as follows: CslA-F: 5'-CGTATGCCTTCTTCGGCTTC-3', CslA-R: 5'TTGTGCGGTCTTTCCAACC-3', GT-F: 5'-TGAACCCAGAGGAGGCTGAC-3', GTR: 5'-GGTGATTTGAAGGGCAATGG-3'. For cyclophilin, which was used as an internal reference gene, we searched for a $C$. japonica homolog using cyclophilin from Chamaecyparis obtusa (Accession number: AB505627) as the query. Based on the results (Cluster ID: CJC00214), the following primer sequences were designed: F: 5'-CGGTGAGTCCATTTACGGTTC-3', R: 5'-TGGTGTTCTTCCCAGCGTTAG-3'. All of the primers were designed using Primer3Plus [20] and synthesized commercially.

\section{Results}

\subsection{Sapling Growth Conditions}

In this study, saplings were grown in either the field or in growth chambers. In both experiments, meteorological equipment was placed beside the saplings to measure temperature, illumination intensity, and ultraviolet intensity (Figure 1). In the field 


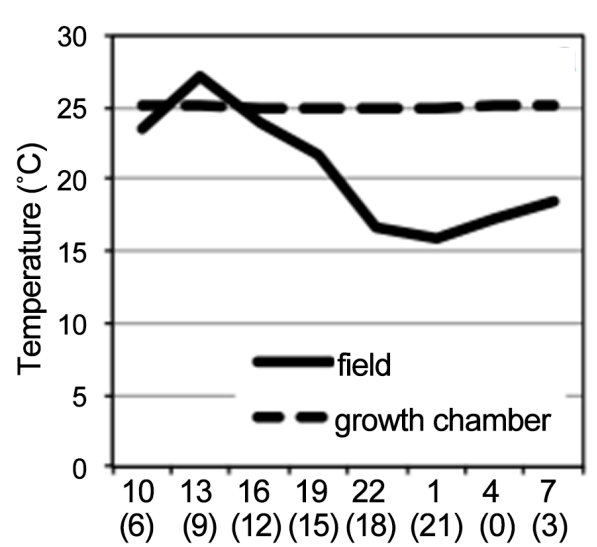

(a)

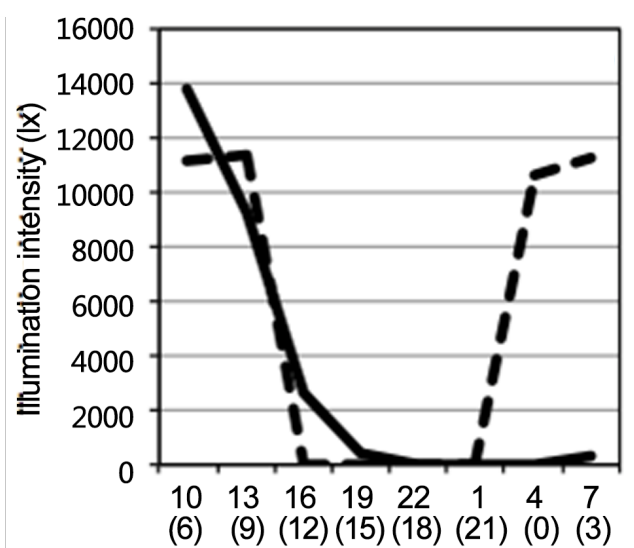

(b)

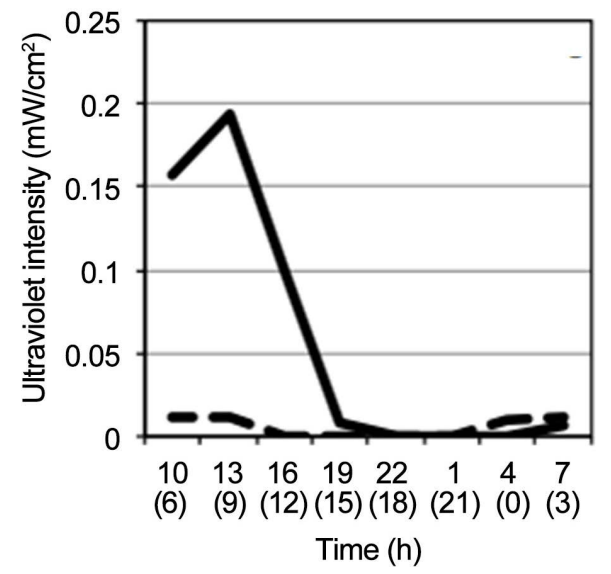

(c)

Figure 1. Growth conditions of $C$. japonica saplings: (a) temperature; (b) illumination intensity; and (c) ultraviolet intensity in the field and growth chamber. On the horizontal axis, the upper line indicates the time in the field experiment and the numbers in parentheses on the lower line indicate hours after the light in the growth chamber switched on. Solid line indicates the field and dashed line indicates the growth chamber.

experiment, temperature was the highest at 13:00 and the lowest at 01:00 (Figure 1(a)). Illumination intensity started to increase around 07:00, peaked at 10:00, and descended to $405 \mathrm{~lx}$ at 19:00 (Figure 1(b)). Ultraviolet intensity showed almost the same trend as illumination intensity; however, the peak occurred at 13:00 (Figure 1(c)). In the growth chamber experiment, the temperature was maintained at $25^{\circ} \mathrm{C}$ (Figure 1(a)). Illumination intensity during the light period $(0-12 \mathrm{~h}$ ) was $\sim 11000 \mathrm{~lx}$, equivalent to that observed at 10:00 and 13:00 in the field (Figure 1(b)). During the light period, weak ultraviolet intensity was observed, comparable to that observed at dawn (07:00) and dusk (19:00) in the field (Figure 1(c)).

\subsection{Diurnal Periodicity of Gene Expression in the Field}

According to electron microscopy observations of the innermost surface of tracheids, cellulose microfibrils deposit during the day and hemicellulose deposits at night [7] 
[10]. In this study, we selected CesA and $S U S$ as representative genes involved in cellulose synthesis and CsIA and GT as representative genes involved in hemicellulose synthesis, and examined the diurnal fluctuations of their transcript abundances (Figure 2). The transcript abundance of CesA was greatest at dawn (04:00 - 07:00) and lowest during the day ( 13:00) (Figure 2(a)). No diurnal periodicity was observed for SUS (Figure 2(b)). The transcript abundance of $C s l A$, involved in glucomannan synthesis, was high from the evening until the morning (16:00 - 07:00) and was lowest around noon (10:00 - 13:00) (Figure 2(c)). The transcript abundance of $G T$, involved in xylan synthesis, showed almost the same pattern as CslA; however, it fluctuated less than CslA (Figure 2(d)).

Next, we examined diurnal fluctuations in the transcript abundance of genes involved in lignin synthesis, which have not been previously revealed by microscopy. We chose eight genes (PAL, C4H, 4CL, HCT, C3H, CCoAOMT, CCR, and CAD) in the phenylpropanoid biosynthetic pathway (Figure 3 ) and examined their diurnal fluctuations in transcript abundance (Figure 4). The transcript abundance of $P A L$, which acts at the starting point of the phenylpropanoid pathway, showed the highest peaks at dusk (16:00 - 19:00) (Figure 4(a), solid line). By adjusting the longitudinal axis of the graph to more easily observe peaks, we noticed small peaks at dawn (04:00 - 07:00) (Figure 4(a), dashed line). C4H peaked at dusk (16:00 - 19:00) and dawn (04:00) with comparable peak heights, while its transcript abundance was lowest at 10:00 (Figure 4(b)). Two types of peaks were observed for $4 C L$, a sharp peak at dusk (16:00 - 19:00) and a moderate peak at dawn (04:00 - 07:00), while its transcript abundance was lowest at

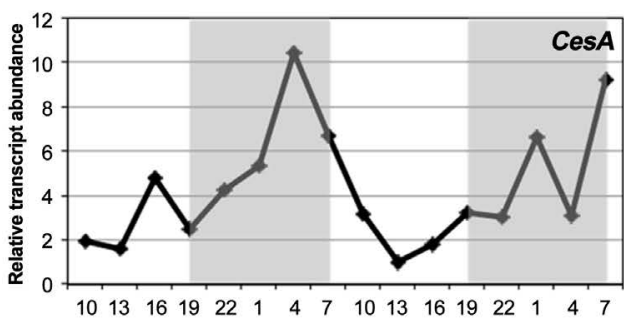

(a)

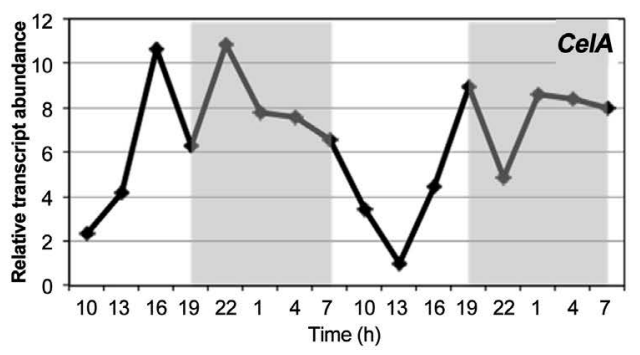

(c)

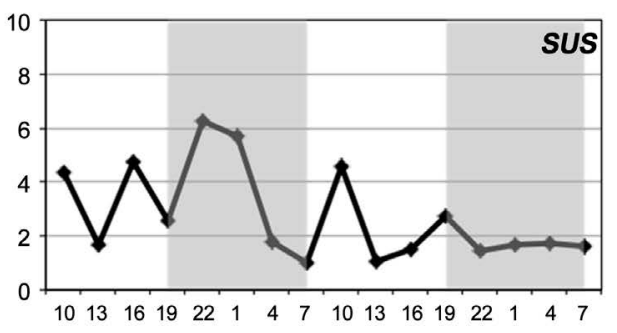

(b)

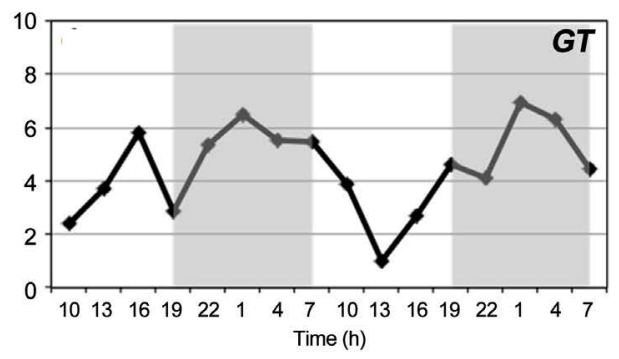

(d)

Figure 2. Diurnal fluctuations in the expression of genes involved in cellulose and hemicellulose synthesis in the field: (a) CesA; (b) SUS; (c) CsIA; and (d) GT. Samples showing the lowest expression for each gene were used as a reference for relative quantification. The gray background color indicates that the illumination intensity was $<500 \mathrm{~lx}$. 


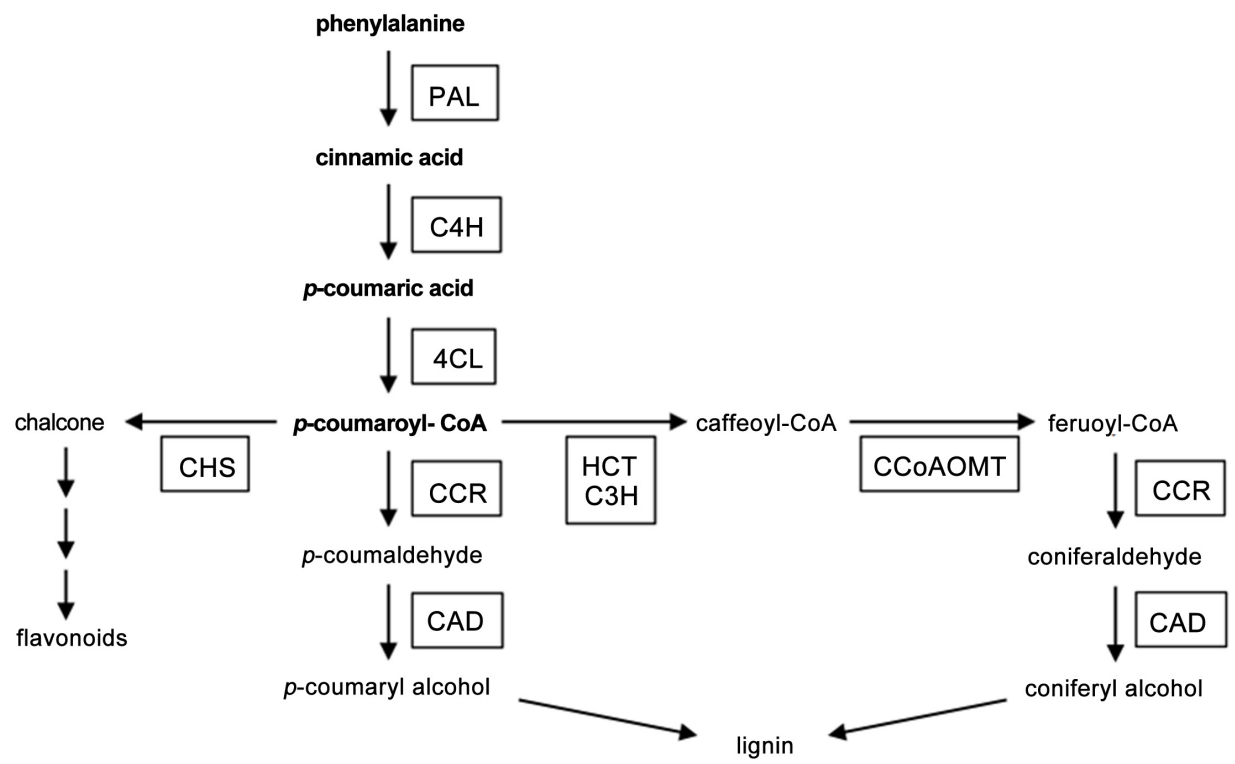

Figure 3. Simplified phenylpropanoid pathway. Bold typed compounds indicate that they are used in both monolignol and flavonoid synthesis. Enzymes working in the pathway are indicated by the boxes.

around noon (10:00 - 13:00) (Figure 4(c)). For HCT, $C 3 H$, and CCoAOMT, peaks of similar size were observed at dusk (16:00 - 19:00) and dawn (04:00), while their transcript abundances were lowest at around noon (10:00 - 13:00) (Figure 4(d)-(f)). Among these three genes, the diurnal fluctuations were clearest for $H C T$, which is located in the upstream portion of the phenylpropanoid pathway (Figure 3), while the periodicity of CCOAOMT, which is located downstream, was the most disturbed. For $C C R$ and $C A D$, we observed slight increases in transcript abundance around dusk (16:00 - 19:00) and decreases during the day (13:00); however, the extent of their fluctuations was smaller than that of the other genes, and diurnal periodicity was not observed (Figure 4(g), Figure 4(h)).

\subsection{Diurnal Periodicity of Gene Expression in the Growth Chambers}

Similar to the field experiments, we examined the diurnal fluctuations of eight genes involved in monolignol biosynthesis in saplings grown in growth chambers under a 12 $\mathrm{h}$ light/dark cycle (Figure 5). We collected samples twice, on 30 May and 6 June. Overall, the extent of the diurnal fluctuations on 6 June was larger than that on 30 May, and the periodicity was easier to recognize. We observed no common patterns between the two sampling days in the diurnal fluctuations of $P A L$, which acts at the starting point of the phenylpropanoid pathway (Figure 5(a)). For $C 4 H$ and $4 C L$, two peaks were observed daily; the first peak was observed 6 - $9 \mathrm{~h}$ after the light switched on and the second peak was observed at the end of the dark period $(\sim 0 \mathrm{~h})$ on both days. In addition, its transcript abundance was lowest during the first half of the dark period (12 - 18 h) (Figure 5(b), Figure 5(c)). For the five remaining genes (HCT, C3H, CCOAOMT, $C C R$, and $C A D)$, two peaks were observed on both days; the first one $6-9 \mathrm{~h}$ after the 


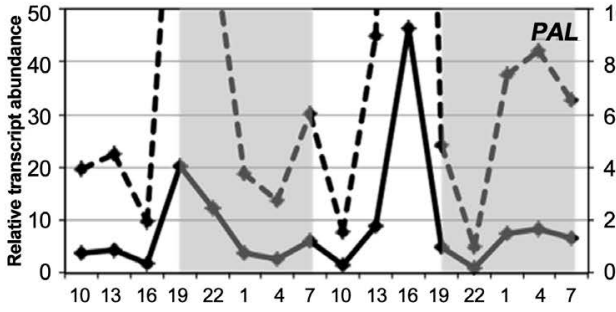

(a)

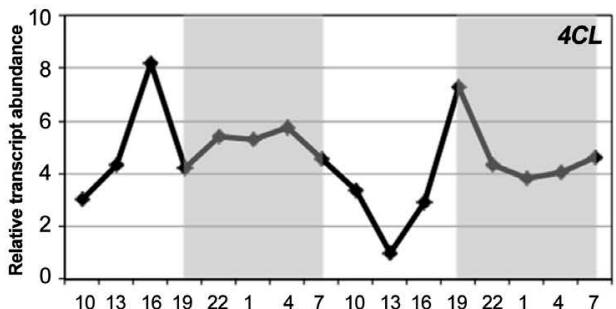

(c)

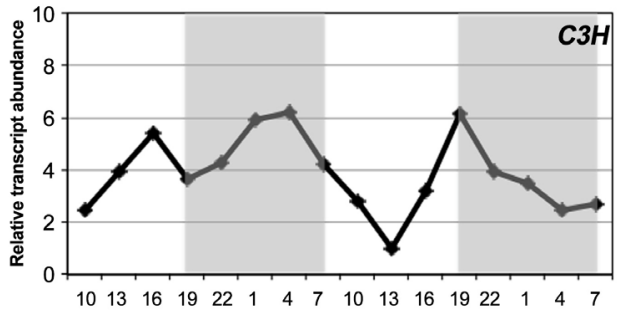

(e)

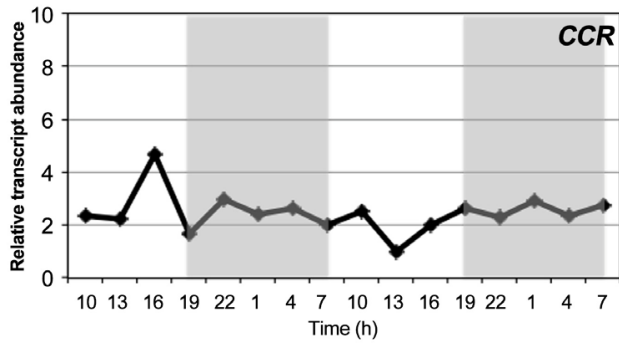

(g)

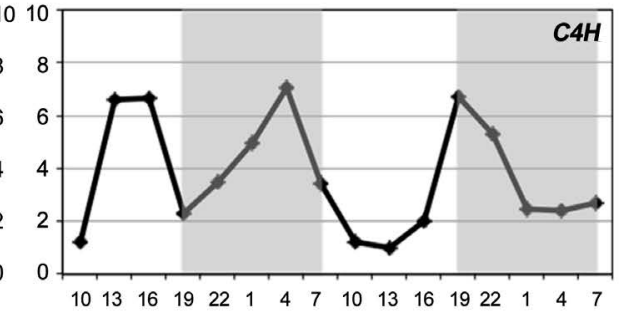

(b)

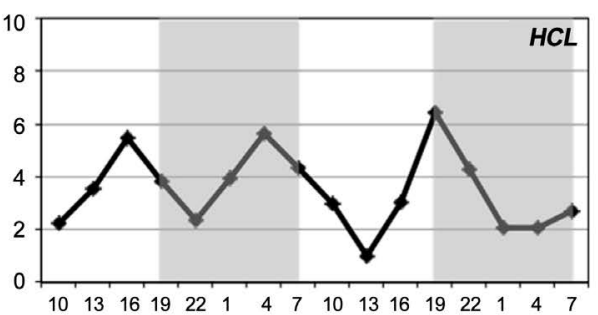

(d)

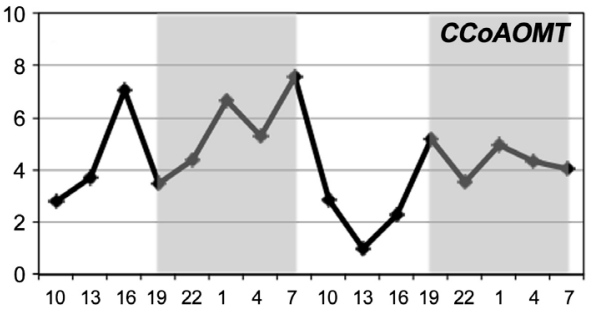

(f)

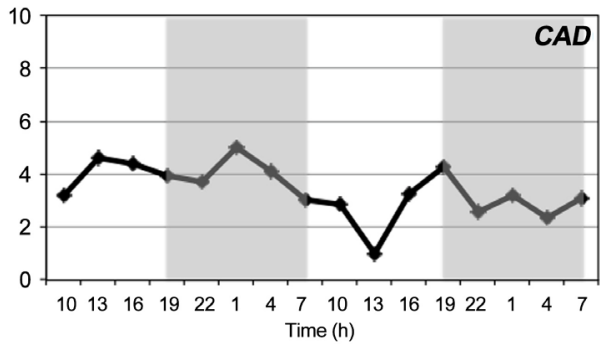

(h)

Figure 4. Diurnal fluctuations in the expression of genes involved in monolignol synthesis in the field: (a) $P A L$; (b) $C A H$; (c) $4 C L$; (d) $H C T$; (e) $C 3 H$; (f) $C C O A O M T$; (g) $C C R$; and (h) $C A D$. Samples showing the lowest expression for each gene were used as a reference for relative quantification. The gray background color indicates that the illumination intensity was $<500 \mathrm{~lx}$. To easily recognize peaks, a second axis, which was adjusted longitudinally, was added to the $P A L$ graph (dashed line).

light switched on and the second one at the end of the dark period $(\sim 0 \mathrm{~h})$. In addition, their transcript abundances were lowest during the first half of the dark period (12 - 18 h) (Figure 5(d)-(h)).

\section{Discussion}

We first examined the diurnal fluctuations in gene expression related to cellulose and 
30 May

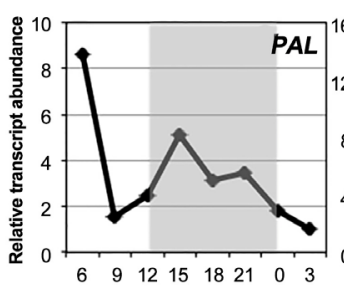

(a)

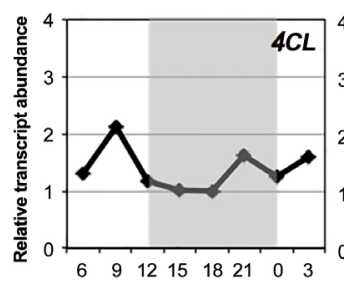

(c)

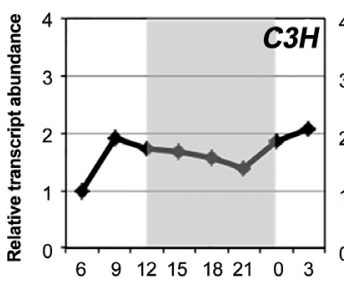

(e)

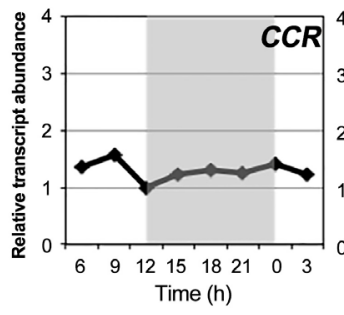

(g)
6 June
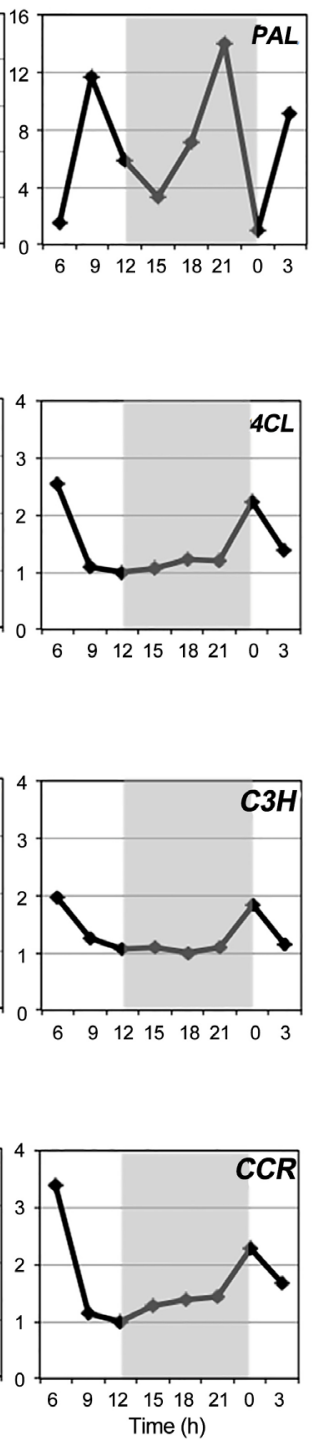

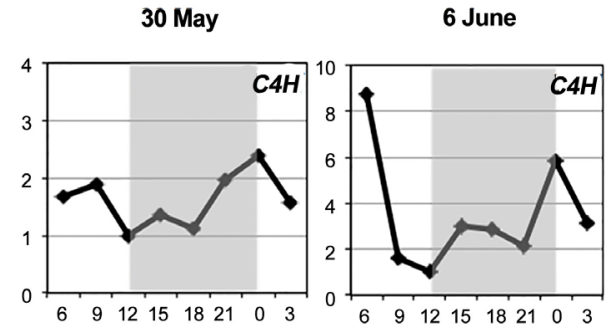

(b)

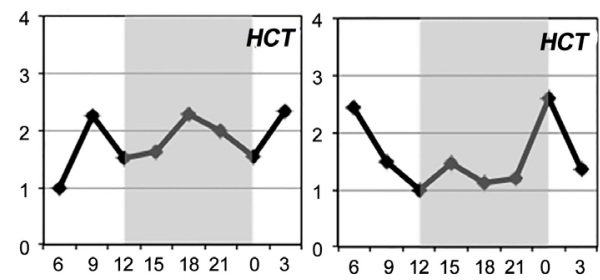

(d)

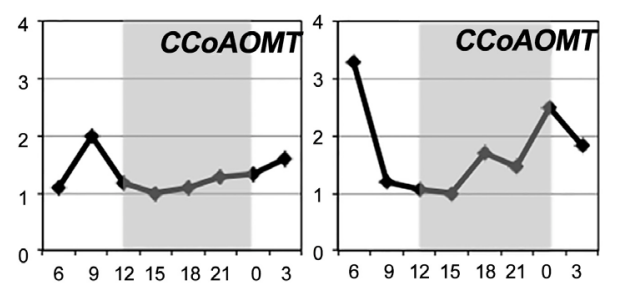

(f)

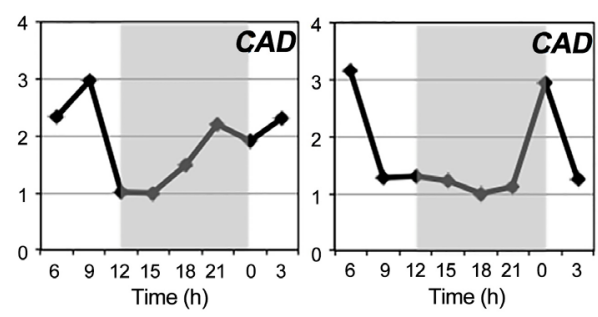

(h)

Figure 5. Diurnal fluctuations in the expression of genes involved in monolignol synthesis in the growth chambers: (a) $P A L$; (b) $C A H$; (c) $4 C L$; (d) HCT; (e) C3H; (f) CCOAOMT; (g) CCR; and (h) $C A D$. The samples were collected in twice: 30 May (left column) and 6 June (right column). The horizontal axis indicates hours after the light in the growth chamber switched on. Samples showing the lowest expression for each gene were used as a reference for relative quantification. The graybackground color indicates the dark period.

hemicellulose synthesis, which had previously been observed by electron microscopy to exhibit periodicity in their deposition on the innermost surface of cell walls. We observed that $\operatorname{Ces} A$ gene expression peaked at dawn (04:00 - 07:00) (Figure 2(a)). Considering that cellulose microfibrils have been observed in the innermost surface of cell walls during the day [8] [9], increasing Ces $A$ gene expression at dawn probably occurs 
in preparation for cellulose synthesis during the day. In contrast, the expression of CsIA and $G T$ genes, which are involved in the main chain syntheses of glucomannan and xylan, respectively, increased at night (16:00 - 07:00) (Figure 2(c), Figure 2(d)). Considering that matrix containing hemicellulose has been observed in the innermost surface of cell walls at night [7] [10], increasing gene expression involved in the syntheses of glucomannan and xylan at night probably aids in the deposition of hemicellulose at night. Because the diurnal fluctuations in the expression of genes involved in cellulose and hemicellulose synthesis were in agreement with the observations of the innermost surfaces of cell walls by electron microscopy, we determined that quantification of gene expression would be a useful tool for studying the diurnal periodicity of lignin deposition not previously revealed via microscopy.

Subsequently, we examined diurnal fluctuations in the expression of eight genes involved in monolignol synthesis using saplings grown in both the field and in growth chambers (Figure 4, Figure 5). From this experiment, we observed four main trends, discussed below. First, when the growth conditions were the same, the graphed wave profiles of the eight genes tended to resemble each other. Genes of the monolignol synthesis pathway are coordinately regulated during transcription [13] [21]. For example, the Myb transcriptional factor is a candidate as a regulator, and genes in the pathway are probably regulated when the Myb transcriptional factor binds to AC elements present in the promoter regions of genes involved in lignin synthesis [22].

Second, the expression of each gene peaked twice a day, at dusk (16:00 - 19:00) and dawn (04:00 - 07:00) in the field experiment, and 6 - 9 and $0 \mathrm{~h}$ after the light switched on in the growth chamber experiment. This can be discussed in terms of the illumination intensity data measured by the meteorological equipment (Figure 1(b)). The first peak appeared in the field and growth chambers at 16:00 - 19:00 and $6-9 \mathrm{~h}$ after the light switched on, respectively; both of these timings corresponded to 6 - $9 \mathrm{~h}$ after the saplings had started to be exposed to intense light $(>10,000 \mathrm{~lx})$. In addition, the second peak appeared in the field and growth chambers at 04:00 - 07:00 and $0 \mathrm{~h}$ after the light switched on, respectively; both of these timings corresponded with switching from a dark to light period. According to these observations, the timings of both peaks had a commonality between the field and growth chambers. Similarly, Rogers et al. [23] and Solomon et al. [14] observed two daily peaks in the diurnal fluctuations of gene expression involved in monolignol synthesis.

Third, in the field experiment, the peaks in $P A L$ and $4 C L$ expression were higher and occurred more sharply at dusk than at dawn. Fourth, in the field experiment, the extent of the diurnal fluctuations was larger for $P A L, C 4 H$, and $4 C L$, which act in the upstream portion of the phenylpropanoid pathway (Figure 3), than for $C C R$ and $C A D$, which function in the downstream portion of the pathway. Both of these phenomena were only observed in the field experiment, and not in the growth chamber experiment. Differences between the field and growth chamber conditions included temperature, length of the light and dark periods, and ultraviolet intensity. The difference in ultraviolet intensity was particularly noticeable (Figure 1(c)). According to Hosoo et al. [9], 
diurnal periodicity in the deposition of cell wall components observed in the innermost surface of the secondary walls was induced not by temperature or time but by light conditions. In addition, from the perspective of the phenylpropanoid pathway (Figure 3), compounds synthesized by $P A L, C 4 H$, and $4 C L$ can become either monolignols or flavonoids, because these three enzymes are positioned upstream of the branching point of monolignol and flavonoid synthesis [24]. Flavonoid concentrations increase when plants are exposed to ultraviolet rays, and have a protective role against ultraviolet radiation [25]. Therefore, an increase in $P A L$ and $4 C L$ for flavonoid synthesis in response to ultraviolet radiation may partially explain the third and fourth observations.

\section{Conclusion}

We examined diurnal fluctuations in the expression of genes involved in cellulose and hemicellulose synthesis that had previously been studied by electron microscopy to determine their deposition periodicities. Based on our results, we found similarities between previous electron microscopy observations of the innermost surface of cell walls and the diurnal fluctuation in gene expression. To gain new insights into the diurnal periodicity of lignin deposition, which have not been revealed by microscopic observations, we evaluated diurnal fluctuations in the expression of genes involved in monolignol biosynthesis and found that expression peaked twice daily, at dusk and dawn. In this study, we focused on genes in the monolignol synthesis pathway as the genes related to lignin synthesis. However, lignin synthesis consists of three steps: monolignol synthesis, transport, and polymerization [26]. Therefore, periodicity in the entire process of lignin deposition should be clarified in future studies by examining diurnal fluctuations in the expression of genes involved in transport and polymerization.

\section{References}

[1] Kimura, S., Laosinchai, W., Itoh, T., Cui, X., Linder, C.R. and Brown, Jr.R.M. (1999) Immunogold Labeling of Rosette Terminal Cellulose-Synthesizing Complexes in the Vascular Plant Vigna angularis. The Plant Cell, 11, 2075-2085. https://doi.org/10.1105/tpc.11.11.2075

[2] Takabe, K., Fujita, M., Harada, H. and Saiki, H. (1981) The Deposition of Cell Wall Components in Differentiating Tracheids of Sugi. Mokuzai Gakkaishi, 27, 249-255.

[3] Northcote, D.H. and Pickett-Heaps, J.D. (1966) A Function of the Golgi Apparatus in Polysaccharide Synthesis and Transport in the Root-Cap Cells of Wheat. Biochemical Journal, 98, 159-167. https://doi.org/10.1042/bj0980159

[4] Harkin, J.M. and Obst, J.R. (1973) Lignification in Trees: Indication of Exclusive Peroxidase Participation. Science Translational Medicine, 180, 296-298.

https://doi.org/10.1126/science.180.4083.296

[5] Bao, W., O'Malley, D.M., Whetten, R. and Sederoff, R.R. (1993) A Laccase Associated with Lignification in Loblolly Pine Xylem. Science, 260, 672-674. https://doi.org/10.1126/science.260.5108.672

[6] Bobák, M. and Nečesaný, V. (1967) Changes in the Formation of the Lignified Cell Wall within a Twenty-Four Hour Period. Biologia Plantarum, 9, 195-201.

https://doi.org/10.1007/bf02929737 
[7] Hosoo, Y., Yoshida, M., Imai, T. and Okuyama, T. (2002) Diurnal Difference in the Amount of Immunogold-Labeled Glucomannans Detected with Field Emission Scanning Electron Microscopy at the Innermost Surface of Developing Secondary Walls of Differentiating Conifer Tracheids. Planta, 215, 1006-1012.

https://doi.org/10.1007/s00425-002-0824-3

[8] Yoshida, M., Hosoo, Y. and Okuyama, T. (2000) Periodicity as a Factor in the Generation of Isotropic Compressive Growth Stress between Microfibrils in Cell Wall Formation during a Twenty-Four Hour Period. Holzforschung, 54, 469-473.

https://doi.org/10.1515/HF.2000.079

[9] Hosoo, Y., Yoshida, M., Imai, T. and Okuyama, T. (2003) Diurnal Differences in the Innermost Surface of the S2 Layer in Differentiating Tracheids of Cryptomeria japonica Corresponding to a Light-Dark Cycle. Holzforschung, 57, 567-573. https://doi.org/10.1515/HF.2003.085

[10] Hosoo, Y., Imai, T. and Yoshida, M. (2006) Diurnal Differences in the Supply of Glucomannans and Xylans to Innermost Surface of Cell Walls at Various Developmental Stages from Cambium to Mature Xylem in Cryptomeria japonica. Protoplasma, 229, 11-19. https://doi.org/10.1007/s00709-006-0190-2

[11] Takabe, K., Fujita, M., Harada, H. and Saiki, H. (1981) Lignification Process of Japanese Black Pine (Pinus thunbergii Parl.) Tracheids. Mokuzai Gakkaishi, 27, 813-820.

[12] Terashima, N., Fukushima, K. and Sano, Y. (1988) Heterogeneity in Formation of Lignin. X. Visualization of Lignification Process in Differentiating Xylem of Pine by Microautoradiography. Holzforschung, 42, 347-350. https://doi.org/10.1515/hfsg.1988.42.6.347

[13] Harmer, S.L., et al. (2000) Orchestrared Transcription of Key Pathways in Arabidopsis by the Circadian Clock. Science, 290, 2110-2113. https://doi.org/10.1126/science.290.5499.2110

[14] Solomon, O.L., Berger, D.K. and Myburg, A.A. (2010) Diurnal and Circadian Patterns of Gene Expression in the Developing Xylem of Eucalyptus Trees. South African Journal of Botany, 76, 425-439. https://doi.org/10.1016/j.sajb.2010.02.087

[15] Livak, K.J. and Schmittgen, T.D. (2001) Analysis of Relative Gene Expression Data Using Real-Time Quantitative PCR and the 2(-Delta Delta C(T)) Method. Methods, 25, 402-408. https://doi.org/10.1006/meth.2001.1262

[16] Mishima, K., et al. (2014) Transcriptome Sequencing and Profiling of Expressed Genes in Cambial Zone and Differentiating Xylem of Japanese Cedar (Cryptomeria japonica). BMC Genomics, 15, 219. https://doi.org/10.1186/1471-2164-15-219

[17] Liepman, A.H., Nairn, C.J., Willats, W.G.T., Sørensen, I., Roberts, A.W. and Keegstra, K. (2007) Functional Genomic Analysis Supports Conservation of Function among Cellulose Synthase-Like a Gene Family Members and Suggests Diverse Roles of Mannans in Plants. Plant Physiology, 143, 1881-1893. https://doi.org/10.1104/pp.106.093989

[18] Brown, D.M., Zhang, Z., Stephens, E., Dupree, P. and Turner, S.R. (2009) Characterization of IRX10 and IRX10-Like Reveal an Essential Role in Glucuronoxylan Biosynthesis in Arabidopsis. The Plant Journal, 57, 732-746. https://doi.org/10.1111/j.1365-313X.2008.03729.x

[19] Wu, A.-M., et al. (2009) The Arabidopsis IRX10 and IRX10-Like Glycosyltransferases Are Critical for Glucuronoxylan Biosynthesis during Secondary Cell Wall Formation. The Plant Journal, 57, 718-731. https://doi.org/10.1111/j.1365-313X.2008.03724.x

[20] Untergasser, A., Nijveen, H., Rao, X., Bisseling, T., Geurts, R. and Leunissen, J.A. (2007) Primer3Plus, an Enhanced Web Interface to Primer3. Nucleic Acids Research, 35, W71W74. https://doi.org/10.1093/nar/gkm306 
[21] Koutaniemi, S., et al. (2007) Expression Profiling of the Lignin Biosynthetic Pathway in Norway Spruce Using EST Sequencing and Real-Time RT-PCR. Plant Molecular Biology, 65, 311-328. https://doi.org/10.1007/s11103-007-9220-5

[22] Zhao, Q. and Dixon, R.A. (2011) Transcriptional Networks for Lignin Biosynthesis: More Complex than We Thought? Trends in Plant Science, 16, 227-233. https://doi.org/10.1016/j.tplants.2010.12.005

[23] Rogers, L.A., et al. (2005) Light, the Circadian Clock, and Sugar Perception in the Control of Lignin Biosynthesis. Journal of Experimental Botany, 56, 1651-1663. https://doi.org/10.1093/jxb/eri162

[24] Ferrer, J.-L., Austin, M.B., Stewart, J.C. and Noel, J.P. (2008) Structure and Function of Enzymes Involved in the Biosynthesis of Phenylpropanoids. Plant Physiology and Biochemistry, 46, 356-370. https://doi.org/10.1016/j.plaphy.2007.12.009

[25] Shirley, B.W. (1996) Flavonoid Biosynthesis: "New" Functions for an "Old" Pathway. Trends in Plant Science, 1, 377-382.

[26] Boerjan, W., Ralph, J. and Baucher, M. (2003) Lignin Biosynthesis. Annual Review of Plant Biology, 54, 519-546. https://doi.org/10.1146/annurev.arplant.54.031902.134938

\section{Submit or recommend next manuscript to SCIRP and we will provide best service} for you:

Accepting pre-submission inquiries through Email, Facebook, LinkedIn, Twitter, etc. A wide selection of journals (inclusive of 9 subjects, more than 200 journals)

Providing 24-hour high-quality service

User-friendly online submission system

Fair and swift peer-review system

Efficient typesetting and proofreading procedure

Display of the result of downloads and visits, as well as the number of cited articles Maximum dissemination of your research work

Submit your manuscript at: http://papersubmission.scirp.org/

Or contact ajps@scirp.org 